\title{
Statins Protect the Blood Brain Barrier Acutely after Experimental Intracerebral Hemorrhage*
}

\author{
Dongmei Yang ${ }^{1 \#}$, Robert A. Knight ${ }^{2,3 \#}$, Yuxia Han $^{1}$, Kishor Karki ${ }^{2,3}$, Jianfeng Zhang ${ }^{1}$, \\ Michael Chopp ${ }^{2,3}$, Donald M. Seyfried ${ }^{1}$ \\ ${ }^{1}$ Departments of Neurosurgery, Henry Ford Health System, Detroit, USA \\ ${ }^{2}$ Departments of Neurology, Henry Ford Health System, Detroit, USA \\ ${ }^{3}$ Departments of Physics, Oakland University, Rochester, USA \\ Email: dseyfri1@hfhs.org
}

Received November 2, 2012; revised December 3, 2012; accepted December 10, 2012

\begin{abstract}
Objectives: The goal of this study was to measure the impact of simvastatin and atorvastatin treatment on blood brain barrier (BBB) integrity after experimental intracerebral hemorrhage (ICH). Methods: Primary ICH was induced in 27 male Wistar rats by stereotactic injection of $100 \mu \mathrm{L}$ of autologous blood into the striatum. Rats were divided into three groups ( $\mathrm{n}=9 /$ group): 1) oral treatment $(2 \mathrm{mg} / \mathrm{kg})$ of atorvastatin, 2) oral treatment $(2 \mathrm{mg} / \mathrm{kg})$ simvastatin, or 3) phosphate buffered saline daily starting 24-hour post-ICH and continuing daily for the next 3 days. On the fourth day, the animals underwent magnetic resonance imaging (MRI) for measurements of $\mathrm{T}_{1 \text { sat }}$ (a marker for BBB integrity), $\mathrm{T}_{2}$ (edema), and cerebral blood flow (CBF). After MRI, the animals were sacrificed and immunohistology or Western blotting was performed. Results: MRI data for animals receiving simvastatin treatment showed significantly reduced BBB dysfunction and improved CBF in the ICH rim compared to controls $(P<0.05) 4$ days after ICH. Simvastatin also significantly reduced edema $\left(\mathrm{T}_{2}\right)$ in the rim at 4 days after ICH $(P<0.05)$. Both statin-treated groups demonstrated increased occludin and endothelial barrier antigen levels within the vessel walls, indicating better preservation of BBB function $(P<0.05)$ and increased number of blood vessels $(P<0.05)$. Conclusions: Simvastatin treatment administered acutely after ICH protects BBB integrity as measured by MRI and correlative immunohistochemistry. There was also evidence of improved CBF and reduced edema by MRI. Conversely, atorvastatin showed a non-significant trend by MRI measurement.
\end{abstract}

Keywords: Intracerebral Hemorrhage; Atorvastatin; Occludin; Simvastatin; Blood Brain Barrier

\section{Introduction}

Blood-brain barrier (BBB) dysfunction following intracerebral hemorrhage (ICH) is assumed to contribute to brain injury [1]. Both animal models and human studies show that BBB disruption occurs acutely after ICH [2-5]. The mechanisms of BBB breakdown that underlie the progression of ICH are only partially known [5], but researchers have shown that BBB disruption increases cerebrovascular permeability, thereby allowing the entrance of potentially neurotoxic compounds and leukocytes into the brain parenchyma which can in turn cause edema formation $[1,4]$. The extent of edema along with the larger hematoma volume correlates with high mortality and poor prognosis after ICH [6]. Current surgical and medical approaches for ICH treatment have been ineffective [7], therefore, a strategy aimed at early BBB protection

*Supported by: National Institute of Health grant RO1 NS05858101 (D.M.S.).

"Both authors contributed equally to this work. after ICH would be a useful therapeutic advance.

Statins or 3-hydroxy-3-methyl-glutaryl-coenzyme A (HMG-CoA) reductase inhibitors are widely employed as potent inhibitors of cholesterol biosynthesis [8]. When administered after ischemic stroke or traumatic brain injury (TBI), these agents have been shown to provide neuroprotection with beneficial effects on the neuronal and neurovascular systems [9-11]. This has been presumed to be due to the capacity of statins to improve or restore endothelial function, enhance angiogenesis and neurogenesis, increase the stability of atherosclerotic plaques, and decrease oxidative stress and vascular inflammation $[8,9]$. In previous laboratory studies, we found that both atorvastatin and simvastatin enhanced functional outcome and promoted vascular recovery 4 weeks after ICH $[12,13]$; however, it is not known if statins can protect the BBB from injury during the early stages of ICH. To test the hypothesis that statins significantly protect $\mathrm{BBB}$ integrity acutely and/or ameliorate the increases in BBB perme- 
ability often noted after ICH, we investigated the early effects of atorvastatin and simvastatin in an experimental ICH model. We also measured other mechanisms that might be responsible for such an effect.

\section{Materials and Methods}

\subsection{Experimental Model}

All experimental procedures were approved by Henry Ford Hospital's Institutional Animal Care and Use Committee (IACUC No. 1061). Twenty-seven adult male Wistar rats $(270$ - $330 \mathrm{~g})$ were anesthetized intraperitoneally with ketamine $(90 \mathrm{mg} / \mathrm{kg})$ and xylazine $(5 \mathrm{mg} / \mathrm{kg})$. They were then subjected to ICH by direct infusion of $100 \mu$ l autologous whole blood into the striatal region adjacent to the subventricular zone (SVZ) [14-16]. After $\mathrm{ICH}$, the animals were randomly assigned to atorvastatin (2 $\mathrm{mg} / \mathrm{kg})$, simvastatin $(2 \mathrm{mg} / \mathrm{kg}$ ) or phosphate-buffered saline (PBS; control) treatment groups ( $\mathrm{n}=9 /$ group). Treatment was given by oral gavage starting 24 -h post$\mathrm{ICH}$ and continued daily for 3 days. Three rats from each group were selected and sacrificed at 4 days after ICH for Western blot. The remaining 6 rats in each group received daily injections of bromodeoxyuridine (BrdU) $100 \mathrm{mg} / \mathrm{kg}$ (Sigma) from 1 to 4 days post-ICH, intraperitoneally.

\subsection{MR Imaging and Analysis}

MRI measurements were performed 4 days after ICH, using a 7 Tesla, 20-cm bore superconducting magnet (Magnex Scientific, Inc. Palo Alto, CA) interfaced to a Bruker Avance console running Paravision 3.0.2 (Bruker Biospin MRI, Billerica, CA) $[13,14]$. The imaging protocol employed used a 32-mm field of view (FOV). Briefly, the protocol included estimates of the following: 1) cerebral blood flow (CBF); 2) spin-spin relaxation times $\left(\mathrm{T}_{2}\right)$; 3) spin-lattice relaxation times measured in the presence of off-resonance saturation of the bound proton signal $\left(\mathrm{T}_{1 \text { sat }}\right)$; and 4) estimates of post-ICH induced changes in the blood-to-brain transfer constant $\left(\mathrm{K}_{\mathrm{i}}\right)$. The $\mathrm{K}_{\mathrm{i}}$ estimates were obtained using an MR contrast agent (Gd-DTPA: $0.2 \mathrm{mmol} / \mathrm{kg}$ body wt) that was administered by bolus injection via a tail vein during sequential MRI measurements.

The CBF estimates were acquired using an arterial spin labeling technique [17]. This technique is based on the selective inversion of inflowing blood water protons at the level of the carotid arteries prior to MRI measurement in the brain. The inversion pulse was applied for $1 \mathrm{~s}$ at a $\mathrm{B}_{1}$ amplitude of $0.3 \mathrm{kHz}$, and had a frequency offset of $\pm 6 \mathrm{kHz}$. It was followed by an SE sequence with TR/ $\mathrm{TE}=1060 \mathrm{~ms} / 40 \mathrm{~ms}$. Four averages of the image were acquired with the gradient polarities and the RF pulse frequency offsets reversed to remove any gradient asymmetries in the axial direction. The labeled slice was located approximately $2 \mathrm{~cm}$ distal to the imaging slice. The imaging slice was $2-\mathrm{mm}$ thick and was acquired using 64 $\times 64$ matrix. Total time for the entire series was $17 \mathrm{~min}$ $55 \mathrm{~s}$.

The $\mathrm{T}_{2}$ estimates were measured using a standard CarrPurcell-Meiboom-Gill (CPMG) multi-slice (13 slices each of 1-mm thickness) multi-echo (6 echoes) MRI sequence. Echo times (TEs) were 15, 30, 45, 60, 75 and $90 \mathrm{~ms}$, and repetition time $(\mathrm{TR})$ was $5.0 \mathrm{~s}$. Images were produced using a $128 \times 64$ matrix.

The $\mathrm{T}_{1 \text { sat }}$ estimates were acquired using an imaging variant of the T-one by multiple readout pulses (TOMROP) sequence $[18,19]$. This was done by inserting two continuous wave $(\mathrm{CW}) \mathrm{RF}$ saturation pulses into the Look-Locker sequence: the first (4.5 s long) immediately before the inversion pulse and the second (40 ms long) after the signal acquisition. The offset frequency of the saturation pulses was $8 \mathrm{kHz}$, and the rotational frequency of the $\mathrm{B}_{1}$ field was $0.5 \mathrm{kHz}$. Initially, the longitudinal magnetization was inverted using an $8 \mathrm{~ms}$ non-selective adiabatic hyperbolic secant pulse. One phase encode line of 32 small-tip angle gradient echo images $(\mathrm{TE}=7.0 \mathrm{~ms}$ ) was acquired at $80-\mathrm{ms}$ intervals after each inversion. With this sequence, a single 2 -mm thick slice $\mathrm{T}_{1 \text { sat }}$ map was obtained in $\sim 9 \min (\mathrm{TR}=8 \mathrm{~s}, 128 \times 64$ matrix $)$.

The MR data were transferred to a Unix-based system for image processing and analysis. All MR images were reconstructed using a $128 \times 128$ matrix. Regions of interest (ROIs) representing hematoma core and adjacent rim were identified by windowing $\mathrm{T}_{2}$ values. All other MRI parameter maps were coregistered to the $T_{2}$ maps. The MRI parameters were measured in these selected ROIs and the corresponding contralateral regions, and are reported as ipsilateral/contralateral ratios.

\subsection{Immunohistochemistry}

All animals were sacrificed 4 days post-ICH following MRI for either Western blot or immunohistochemical analysis. Endothelial barrier antigen (EBA) (1:1000 dilution; Sternberger Monoclonals, Baltimore, MD), and occludin (1:200 dilution; Invitrogen, Carlsbad, CA) immunostaining were performed as described previously [16]. All immunostainings were performed at the same time with two negative controls (i.e., the omission of primary antibody and the use of pre-immune serum) for quality control of the immunoassaying procedure. To determine whether BrdU-immunoreactive endothelial cells express EBA, double immunohistochemical staining was used to identify BrdU (1:100 dilution; Boehringer, Indianapolis, IN) with the endothelial marker. The tissues were counterstained with $0.1 \mathrm{mg} / \mathrm{ml}$ DAPI (Sigma, St. Louis, MO) 
in PBS for $5 \mathrm{~min}$ at room temperature.

For quantitative measurements of occludin and EBA, 6 immunostained coronal sections and 8 fields of view from the striatum in each section were digitized under a $20 \times$ objective (Olympus BX40) using a 3-color CCD video camera (Sony DXC-970MD) interfaced with an MCID image analysis system (Imaging Research). The data are presented as a percentage of positive occludin immunoreactivity area in the border and the average vessel number per square $\mathrm{mm}$. The proportions of BrdU+ endothelial cells were calculated based on the total number of BrdU+ endothelial cells (EBA+/BrdU+/DAPI + ) and the total number of endothelial cells (EBA+/DAPI +$)$ in 20 vessels adjacent to the hematoma from each rat.

\subsection{Western Blots}

To confirm the immunostaining data and to measure occludin expression, Western blot assays were performed. Homogenates of tissue samples taken from the border zone around the hematoma were obtained at 4 days after $\mathrm{ICH}$. The protein concentrations of extracts were tested using a BCA protein assay reagent kit (Pierce, Rockford, IL). Equal amounts of protein for each group were assayed by SDS/PAGE and transferred to PVDF membranes. The blots were developed with enhanced chemiluminescence (Pierce), digitally scanned (GS-700, BioRad), and analyzed (Molecular analystR, Bio-Rad). Anti$\beta$-actin antibody (Santa Cruz, Santa Cruz, CA) was used as a control.

\subsection{Statistical Analysis}

An analysis of variance (ANOVA) procedure was used to evaluate the ipsilateral/contralateral values of MR parameters at 4 days post-ICH and the results of the immunohistological measures of EBA and occludin expressions between statin-treated and control groups. Data are reported as mean \pm standard error of the mean (SEM). Statistical significance was inferred at $P \leq 0.05$. All measurements were performed by observers blinded to individual treatments.

\section{Results}

\subsection{MR Imaging}

Representative $\mathrm{CBF}, \mathrm{T}_{2}, \mathrm{~T}_{1 \mathrm{sat}}$ maps from control, atorvastatin- and simvastatin-treated animals at 4 days post$\mathrm{ICH}$ are shown in Figure 1. The CBF maps indicate that treatment with statins increased blood flow particularly along the periphery or outer boundary of the central core region when compared to controls. Quantitative analysis showed that the ipsilateral/contralateral $\mathrm{CBF}$ ratios in the rim significantly increased after simvastatin treatment (Figure 2). The control group $\mathrm{T}_{2}$ maps showed a bright

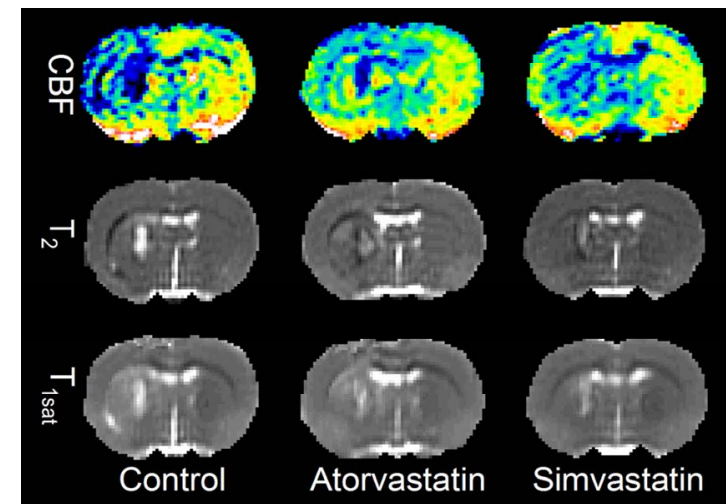

Figure 1. Representative cerebral blood flow (CBF), $T_{2}$, $T_{1 \text { sat }}$ maps obtained from control (left panel), atorvastatintreated (middle panel), and simvastatin-treated animals (right panel) at 4 days post-ICH. The CBF maps (upper panel) indicate treatment with statins increases blood flow particularly in the central core region when compared with control. The $T_{2}$ maps (center panels) in control group show a bright central core region (high $T_{2}$ ) and adjacent surrounding dark rim (low $T_{2}$ ). Conversely, $T_{2}$ results for the statin-treated animals showed a less intense response in the core region (i.e. lower $T_{2}$ values) relative to the rim area than seen in control animals. The $T_{1 \text { sat }}$ maps (lower panels) show the decreased BBB permeability in the core region of the statin-treated animals.

central core region (high $\mathrm{T}_{2}$ ) and an adjacent surrounding dark rim (low $\mathrm{T}_{2}$ ). In comparison, the $\mathrm{T}_{2}$ results for the statin-treated animals showed a less intense response in the core region (i.e. lower $\mathrm{T}_{2}$ values) relative to the rim area than in control animals. Finally, the $\mathrm{T}_{1 \mathrm{sat}}$ maps showed decreased $\mathrm{T}_{1 \text { sat }}$ values in the $\mathrm{ICH}$ border region of the statin-treated animals indicative of lower BBB permeability relative to control rats. Additionally, statistical analysis demonstrated that the ipsilateral/contralateral $\mathrm{T}_{2}$ and $T_{1 \text { sat }}$ ratios significantly decreased in the rim after simvastatin treatment relative to controls (Figure 2). These findings suggest that simvastatin increases $\mathrm{CBF}$, decreases edema and modulates BBB permeability during the acute phase of $\mathrm{ICH}$. Although atorvastatin treatment showed trends dissimilar to simvastatin treatment in its effects, the differences were not significant.

\subsection{Occludin Expression}

To clarify how statins may protect the BBB after ICH, the tight junction protein occludin was studied by immunofluorescent staining and immunoblotting 4 days after ICH. Occludin was expressed in the intima of cerebral capillaries and was dramatically increased in the boundary area around the hematoma after both statin treatments (Figure 3). In agreement with immunohistochemistry results, Western blotting showed that the expression level of occludin in the border zone of animals in both statin-treated groups increased at the designated time 
point, when compared to controls (Figure 4).

\subsection{Angiogenesis}

EBA immunohistochemical staining provides a sensitive and reliable index for cerebral vessels [20]. Angiogenesis was observed after brain injury, and characterized by enlarged vascular perimeters and capillaries sprouting from preexisting blood vessels as well as increased microvessel density and newly formed endothelial cells. In the current study, the boundary area around the hematoma in both statin-treated groups showed an up-regulation in the intensity of immunoreactivity to EBA (Figure 5). The distribution of EBA immunoreactivity in other regions of statin-treated brain tissue appeared similar to that of control animals. A significant increase in the number of cells co-stained with EBA and BrdU was also observed in the same area in statin-treated animals, when compared to control animals (Figure 6).

\section{Discussion}

The present study demonstrates that simvastatin treatment significantly protects $\mathrm{BBB}$ integrity, reduces edema and improves $\mathrm{CBF}$ as measured by MRI during the acute phase after experimental ICH. Atorvastatin showed a non-significant trend by MRI measurement, although both statins induced increased expression of the tight junction protein occludin in the boundary zone. This study also revealed that both statins can promote angiogenesis as early as 4 days after ICH.

More detailed experimental and clinical evidence continues to accumulate regarding the efficacy of statins for treatment of ICH. An earlier study indicated that $2 \mathrm{mg} / \mathrm{kg}$ atorvastatin significantly reduced neurological deficits at 2 weeks to 4 weeks after experimental $\mathrm{ICH}$, while higher doses of $8 \mathrm{mg} / \mathrm{kg}$ did not improve functional outcome or lessen brain damage [15]. In a collagenase ICH model,

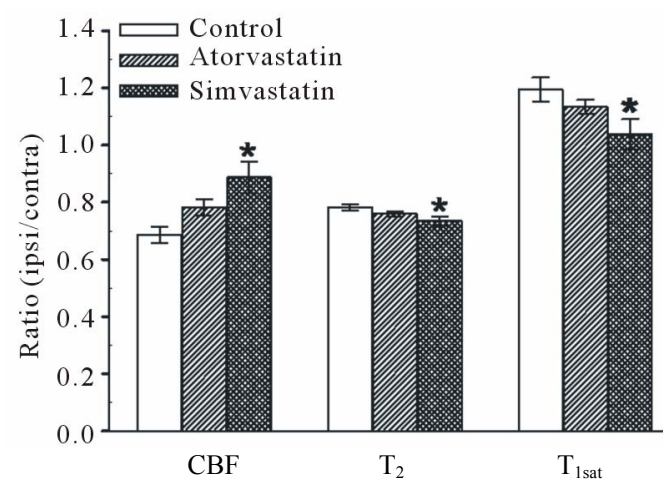

Figure 2. Plots of ipsilateral/contralateral ratio values for cerebral blood flow (CBF), T1sat and T2 at 4 days after intracerebral hemorrhage (ICH) for control, atorvastatinand simvastatin-treated rats. The data indicate that treatment with simvastatin significantly increased CBF, and decreased $\mathrm{T} 1$ sat and $\mathrm{T} 2$ values in the rim compared with the control group. ${ }^{*} \boldsymbol{P}<\mathbf{0 . 0 5}$.
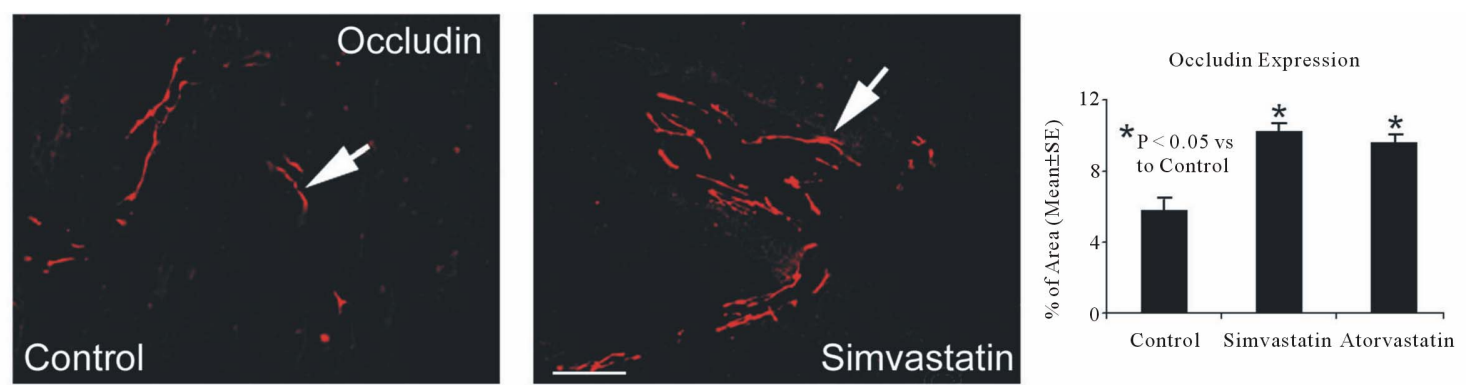

Figure 3. Effects of statin on the tight junction protein occludin. The left and middle panels show occludin immuno-reactivity in the ICH boundary area of representative rats treated with PBS and simvastatin, respectively. The right panel shows quantitative estimates of occluding-positive cells expressed as a percentage of area in the ICH boundary (mean \pm SEM) zone for all three groups. Both statin-treated groups showed significant increases in occludin expression compared to controls $\left({ }^{*} P<0.05\right)$.
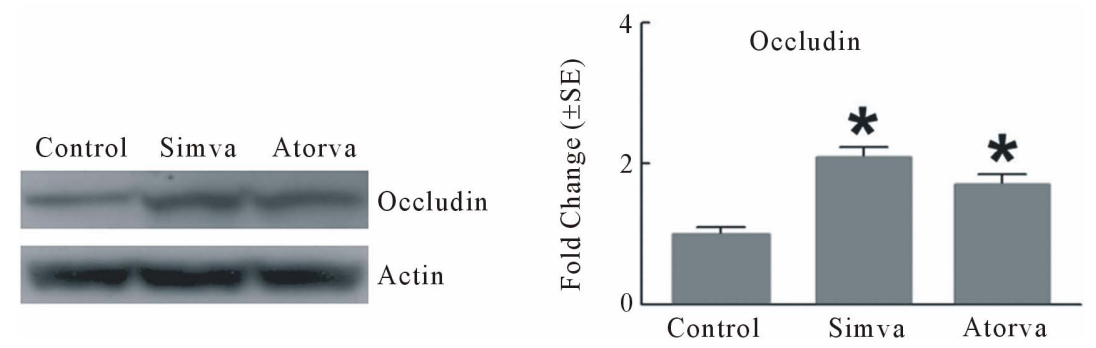

Figure 4. Western blot data are shown for tissue samples taken from the ICH border zone of control and statin-treated animals. Occludin protein expression in the hemorrhagic brain tissue endothelial cells was increased in the statin-treated rats compared to controls. 
Jung et al., found that $1 \mathrm{mg} / \mathrm{kg}$ or $10 \mathrm{mg} / \mathrm{kg}$ atorvastatin promoted sensorimotor recovery after 2 weeks and the effects persisted up to 4 weeks [21]. Several retrospective and prospective studies indicated that the ICH patients on statins had better outcome in comparison to those without statins [22-26]. While the study of the Stroke Prevention by Aggressive Reduction in Cholesterol Levels (SPARCL) demonstrated an increased risk of ICH in patients treated with high doses of atorvastatin, the overall effect was deemed to be one of clinical benefit [27]. Our present result shows that treatment with $2 \mathrm{mg} / \mathrm{kg}$ /day statin for 4 days protected the BBB after ICH, while expansion of the hematoma was not observed by either MRI or histology. These data suggest that a low dose of statin during the acute phase of ICH might be optimal to achieve therapeutic effects without secondary hemorrhage.

The BBB after ICH is disrupted by perihematomal injury and the subsequent inflammatory cascades initiated by coagulation products and toxic blood breakdown products [1]. The autologous whole blood ICH model has been shown to mimic the mechanism of $\mathrm{BBB}$ disruption [28]. The onset of BBB dysfunction was observed to occur at $12 \mathrm{~h}$ to $48 \mathrm{~h}$ after ICH in this model [2]. The current study demonstrated that statin treatment starting $24 \mathrm{~h}$ post-ICH and persisting through Day 4 attenuated brain edema formation and BBB permeability. The post-ICH intervention with statins provided rapid BBB protection; this ultimately may alleviate intracranial complications and promote the improved functional outcome which is observed in these experimental models.

The immunohistochemistry portion of the study supports the concept of endothelial cell-mediated function of the BBB integrity. Tight junctions linking the cerebral endothelial cells play a vital role in BBB function by limiting diffusion of compounds from the systemic circulation to brain parenchyma [29]. Occludin is one of the important transmembrane proteins of tight junctions that are essential for maintenance of the BBB integrity [30].
Decreased expression of occludin correlated with disrupted BBB function in neurological injuries [31,32]. Our results indicated that animals treated with statins after ICH have elevated occludin protein levels relative to controls, which is associated with improved BBB integrity. It is unclear, however, whether the increased protein level is due to enhanced occludin synthesis or its protection from breakdown.

Angiogenesis can begin at $12 \mathrm{~h}$ to $24 \mathrm{~h}$ after experimental ischemic stroke, and clinical data suggest that it is present three to four days after ischemic stroke [33,34]. In parallel, our study showed that after 4 days of statin treatment the number of vessels and proliferating endothelial cells were increased in the boundary zone around
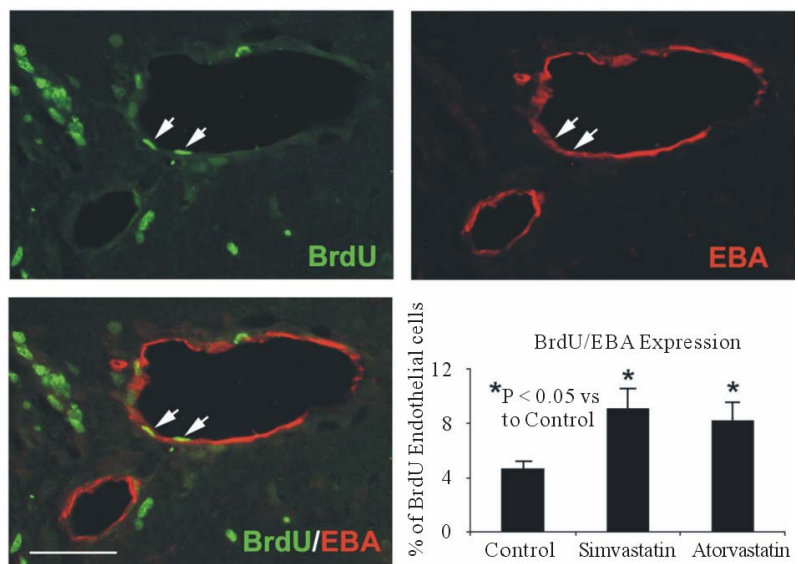

Figure 5. Analysis for proliferating endothelial cells. The upper panels show BrdU (left) and EBA (right) immunostaining. Merged BrdU and EBA images are presented in the lower left panel, showing colocalization of BrdU-EBA for a subpopulation of cells located near the injured site for a simvastatin-treated animal. Arrows indicate the cells that stained positively for both BrdU and EBA. Quantitative measures of the percentage of BrdU-positive endothelial cells (mean \pm SEM) are presented as bar graphs in the lower right panel. The statin-treated rats showed significant increases in BrdU expression compared to controls ( ${ }^{*} P<$ 0.05).
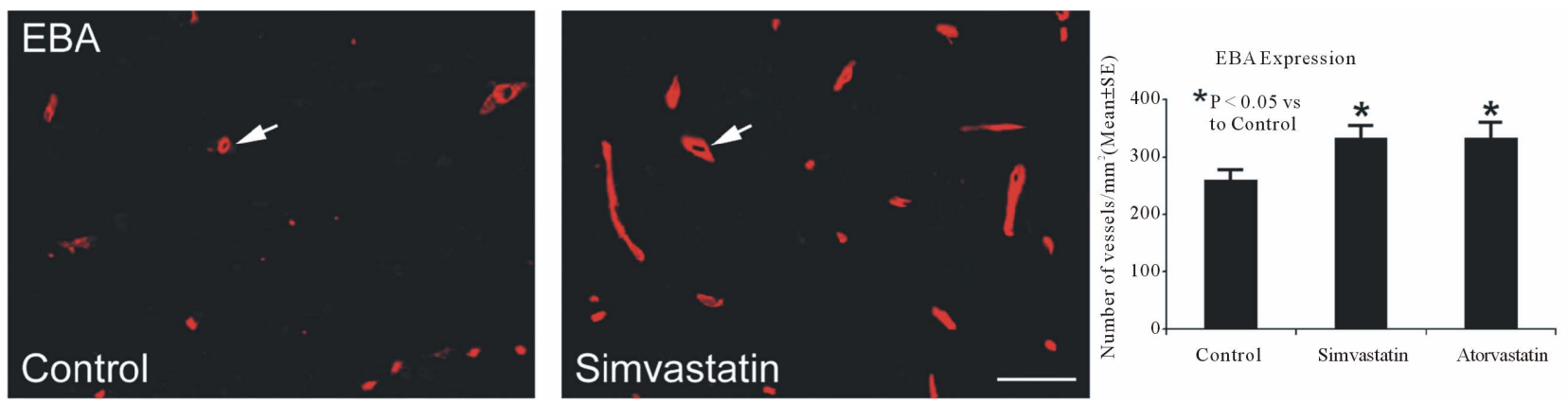

Figure 6. Effects of simvastatin on the blood-brain barrier in the ICH boundary zone. The left and middle panels show EBA immunoreactivity in the ICH boundary area of representative rats treated with PBS and simvastatin, respectively. The right panel shows quantitative measures of EBA immunoreactive vessels for all three groups with significant increases in EBA expression in the statin-treated animals as compared to controls $\left({ }^{*} P<0.05\right)$. 
the hematoma suggesting that angiogenesis can occur as early as 4 days after ICH. We reason that these increased CBF levels observed by MRI may be attributed partially to ongoing angiogenesis.

Our previous study demonstrated that both simvastatin and atorvastatin provided similar neuroprotective and neurorestorative effects at 4 weeks post-ICH [12]. Nevertheless, current MRI studies suggest that at the same dose simvastatin-based therapy was more effective than atorvastatin-based therapy in achieving acute efficacy via $\mathrm{BBB}$ protection or improving $\mathrm{CBF}$ at 4 days post-ICH. The differences between the two statins could be due to their intrinsic differences in plasma life and permeability across the BBB with simvastatin having greater lipophilic properties $[35,36]$. Whether higher doses or longer administration times would render atorvastatin as effective as simvastatin is unknown and requires further study.

In conclusion, simvastatin demonstrated therapeutic potential in acute treatment of ICH as seen in this experimental model. Increased expression of tight junction proteins and the early observation of angiogenesis may represent important mechanisms for the efficacy of simvastatin in $\mathrm{ICH}$.

\section{Acknowledgements}

We would like to thank Susan E. MacPhee-Gray for editorial assistance.

\section{REFERENCES}

[1] R. F. Keep, J. Xiang, S. R. Ennis, A. Andjelkovic, Y. Hua, G. Xi, et al., "Blood-Brain Barrier Function in Intracerebral Hemorrhage," Acta Neurochirurgica Supplement, Vol. 105, 2008, pp. 73-77. doi:10.1007/978-3-211-09469-3 15

[2] G. Y. Yang, A. L. Betz, T. L. Chenevert, J. A. Brunberg and J. T. Hoff, "Experimental Intracerebral Hemorrhage: Relationship between Brain Edema, Blood Flow, and Blood-Brain Barrier Permeability in Rats," Journal of Neurosurgery, Vol. 81, No. 1, 1994, pp. 93-102. doi:10.3171/jns.1994.81.1.0093

[3] R. Thiex and S. E. Tsirka, "Brain Edema after Intracerebral Hemorrhage: Mechanisms, Treatment Options, Management Strategies, and Operative Indications," Neurosurgical Focus, Vol. 22, No. 5, 2007, p. E6. doi:10.3171/foc.2007.22.5.7

[4] K. R. Lee, N. Kawai, S. Kim, O. Sagher and J. T. Hoff, "Mechanisms of Edema Formation after Intracerebral Hemorrhage: Effects of Thrombin on Cerebral Blood Flow, Blood-Brain Barrier Permeability, and Cell Survival in a Rat Model," Journal of Neurosurgery, Vol. 86, No. 2, 1997, pp. 272-278. doi:10.3171/jns.1997.86.2.0272

[5] Z. Karwacki, P. Kowianski, M. Witkowska, M. Karwacka, J. Dziewiatkowski and J. Morys, "The Pathophysiology of Intracerebral Haemorrhage," Folia Morphologica, Vol. 65, No. 4, 2006, pp. 295-300.
[6] G. Y. Chang, "Hematoma Growth Is a Determinant of Mortality and Poor Outcome after Intracerebral Hemorrhage," Neurology, Vol. 68, No. 6, 2007, pp. 471-472.

[7] J. Broderick, S. Connolly, E. Feldmann, D. Hanley, C. Kase, D. Krieger, M. May-berg, L. Morgenstern, C. S. Ogilvy, P. Vespa and M. Zuccarrello, "Guidelines for the Management of Spontaneous Intracerebral Hemorrhage in Adults: 2007 Update: A Guideline from the American Heart Association/American Stroke Association Stroke/ Council, High Blood Pressure Research Council, and the Quality of Care and Outcomes in Research Interdisciplinary Working Group," Circulation, Vol. 116, No. 16, 2007, pp. e391-e413. doi:10.1161/CIRCULATIONAHA.107.183689

[8] M. Takemoto and J. K. Liao, "Pleiotropic Effects of 3-hydroxy-3-methylglutaryl Co-Enzyme a Reductase Inhibitors," Arteriosclerosis, Thrombosis, and Vascular Biology, Vol. 21, No. 11, 2001, pp. 1712-1719. doi:10.1161/hq1101.098486

[9] J. Chen, Z. G. Zhang and Y. Li, "Statins Induce Angiogenesis, Neurogenesis, and Synaptogenesis after Stroke," Annals of Neurology, Vol. 53, No. 6, 2003, pp. 743-751. doi:10.1002/ana.10555

[10] E. F. Wible and D. T. Laskowitz, "Statins in Traumatic Brain Injury," Neurotherapeutics, Vol. 7, No. 1, 2010, pp. 62-73. doi:10.1016/j.nurt.2009.11.003

[11] H. Wu, D. Lu, H. Jiang, Y. Xiong, C. Qu, B. Li, et al., "Simvastatin-Mediated Upregulation of vegf and bdnf, Activation of the pi3k/akt Pathway, and Increase of Neurogenesis Are Associated with Therapeutic Improvement after Traumatic Brain Injury," Journal of Neurotrauma, Vol. 25, No. 2, 2008, pp. 130-139. doi:10.1089/neu.2007.0369

[12] D. Yang, R. A. Knight, Y. Han, K. Karki, J. Zhang, C. Ding, et al., "Vascular Recovery Promoted by Atorvastatin and Simvastatin after Experimental Intracerebral Hemorrhage: Magnetic Resonance Imaging and Histological Study," Journal of Neurosurgery, Vol. 114, No. 4, 2011, pp. 1135-1142. doi:10.3171/2010.7.JNS10163

[13] K. Karki, R. A. Knight, Y. Han, D. Yang, J. Zhang, K. A. Ledbetter, et al., "Simvastatin and Atorvastatin Improve Neurological Outcome after Experimental Intracerebral Hemorrhage," Stroke, Vol. 40, No. 10, 2009, pp. 33843389. doi:10.1161/STROKEAHA.108.544395

[14] R. A. Knight, Y. Han, T. N. Nagaraja, P. Whitton, J. Ding, M. Chopp, et al., "Temporal MRI Assessment of Intracerebral Hemorrhage in Rats," Stroke, Vol. 39, No. 9, 2008, pp. 2596-2602. doi:10.1161/STROKEAHA.107.506683c

[15] D. Seyfried, Y. Han, D. Lu, J. Chen, A. Bydon and M. Chopp, "Improvement in Neurological Outcome after Administration of Atorvastatin Following Experimental Intracerebral Hemorrhage in Rats," Journal of Neurosurgery, Vol. 101, No. 1, 2004, pp. 104-107. doi:10.3171/jns.2004.101.1.0104

[16] D. M. Seyfried, Y. Han, D. Yang, J. Ding, L. H. Shen, S. Savant-Bhonsale, et al., "Localization of Bone Marrow Stromal Cells to the Injury Site after Intracerebral Hemorrhage in Rats," Journal of Neurosurgery, Vol. 112, No. 


\section{2, 2010, pp. 329-335. doi:10.3171/2009.2.JNS08907}

[17] D. S. Williams, J. A. Detre, J. S. Leigh and A. P. Koretsky, "Magnetic Resonance Imaging of Perfusion Using Spin Inversion of Arterial Water," Proceedings of the National Academy of Sciences USA, Vol. 89, No. 1, 1992, pp. 212-216. doi:10.1073/pnas.89.1.212

[18] J. R. Ewing, Q. Jiang, M. Boska, Z. G. Zhang, S. L. Brown, G. H. Li, et al., "T1 and Magnetization Transfer at 7 Tesla in Acute Ischemic Infarct in the Rat," Magnetic Resonance in Medicine, Vol. 41, No. 4, 1999, pp. 696705.

doi:10.1002/(SICI)1522-2594(199904)41:4<696::AID-M RM7>3.0.CO;2-5

[19] G. Brix, L. R. Schad, M. Deimling and W. J. Lorenz, "Fast and Precise t1 Imaging Using a Tomrop Sequence," Magnetic Resonance Imaging, Vol. 8, No. 4, 1990, pp. 351-356. doi:10.1016/0730-725X(90)90041-Y

[20] B. Lin and M. D. Ginsberg, "Quantitative Assessment of the Normal Cerebral Microvasculature by Endothelial Barrier Antigen (EBA) Immunohistochemistry: Application to Focal Cerebral Ischemia," Brain Research, Vol. 865 , No. 2, 2000, pp. 237-244. doi:10.1016/S0006-8993(00)02228-9

[21] K. H. Jung, K. Chu, S. W. Jeong, S. Y. Han, S. T. Lee, J. Y. Kim, et al., "Hmgcoa Reductase Inhibitor, Atorvastatin, Promotes Sensorimotor Recovery, Suppressing Acute Inflammatory Reaction after Experimental Intracerebral Hemorrhage," Stroke, Vol. 35, No. 7, 2004, pp. 17441749. doi:10.1161/01.STR.0000131270.45822.85

[22] N. S. Naval, T. A. Abdelhak, N. Urrunaga, P. Zeballos and M. A. Mirski, "An Association of Prior Statin Use with Decreased Perihematomal Edema," Neurocritical Care, Vol. 8, No. 1, 2008, pp. 13-18. doi:10.1007/s12028-007-0081-1

[23] N. S. Naval, T. A. Abdelhak, P. Zeballos, N. Urrunaga and M. A. Mirski, "Prior Statin Use Reduces Mortality in Intracerebral Hemorrhage," Neurocritical Care, Vol. 8, No. 1, 2008, pp. 6-12. doi:10.1007/s12028-007-0080-2

[24] E. FitzMaurice, L. Wendell, R. Snider, K. Schwab, R. Chanderraj, C. Kinnecom, et al., "Effect of Statins on Intracerebral Hemorrhage Outcome and Recurrence," Stroke, Vol. 39, No. 7, 2008, pp. 2151-2154. doi:10.1161/STROKEAHA.107.508861

[25] H. Tapia-Perez, M. Sanchez-Aguilar, J. G. Torres-Corzo, I. Rodriguez-Leyva, D. Gonzalez-Aguirre, A. GordilloMoscoso, et al., "Use of Statins for the Treatment of Spontaneous Intracerebral Hemorrhage: Results of a Pilot Study," Central European Neurosurgery, Vol. 70, No. 1, 2009, pp. 15-20. doi:10.1055/s-0028-1082064

[26] R. Eichel, S. T. Khoury, T. Ben-Hur, M. Keidar, R. Paniri and R. R. Leker, "Prior Use of Statins and Outcome in Patients with Intracerebral Haemorrhage," European Jour- nal of Neurology, Vol. 17, No. 1, 2010, pp. 78-83. doi:10.1111/j.1468-1331.2009.02747.x

[27] L. B. Goldstein, P. Amarenco, M. Szarek, A. Callahan III, M. Hennerici, H. Sille-sen, et al., "Hemorrhagic Stroke in the Stroke Prevention by Aggressive Reduction in Cholesterol Levels Study," Neurology, Vol. 70, No. 24, 2008, pp. 2364-2370. doi:10.1212/01.wnl.0000296277.63350.77

[28] C. L. MacLellan, G. Silasi, C. C. Poon, C. L. Edmundson, R. Buist, J. Peeling, et al., "Intracerebral Hemorrhage Models in Rat: Comparing Collagenase to Blood Infusion," Journal of Cerebral Blood Flow \& Metabolism, Vol. 28, No. 3, 2008, pp. 516-525. doi:10.1038/sj.jcbfm.9600548

[29] E. Steed, M. S. Balda and K. Matter, "Dynamics and Functions of Tight Junctions," Trends in Cell Biology, Vol. 20, No. 3, 2010, pp. 142-149. doi:10.1016/j.tcb.2009.12.002

[30] M. Furuse, T. Hirase, M. Itoh, A. Nagafuchi, S. Yonemura, S. Tsukita, et al., "Occludin: A Novel Integral Membrane Protein Localizing at Tight Junctions," Journal of Cell Biology, Vol. 123, No. 6, 1993, pp. 17771788. doi:10.1083/jcb.123.6.1777

[31] S. J. Bolton, D. C. Anthony and V. H. Perry, "Loss of the Tight Junction Proteins Occludin and Zonula Occludens1 from Cerebral Vascular Endothelium during Neutrophil-Induced Blood-Brain Barrier Breakdown in Vivo," Neuroscience, Vol. 86, No. 4, 1998, pp. 1245-1257. doi:10.1016/S0306-4522(98)00058-X

[32] R. C. Brown and T. P. Davis, "Hypoxia/Aglycemia Alters Expression of Occludin and Actin in Brain Endothelial Cells," Biochemical and Biophysical Research Communications, Vol. 327, No. 4, 2005, pp. 1114-1123. doi:10.1016/j.bbrc.2004.12.123

[33] J. Krupinski, J. Kaluza, P. Kumar, S. Kumar and J. M. Wang, "Role of Angiogenesis in Patients with Cerebral Ischemic Stroke," Stroke, Vol. 25, No. 9, 1994, pp. 1794 1798. doi:10.1161/01.STR.25.9.1794

[34] T. Hayashi, N. Noshita, T. Sugawara and P. H. Chan, "Temporal Profile of Angiogenesis and Expression of Related Genes in the Brain after Ischemia," Journal of Cerebral Blood Flow \& Metabolism, Vol. 23, No. 2, 2003, pp. 166-180. doi:10.1097/00004647-200302000-00004

[35] M. Endres and U. Laufs, "Effects of Statins on Endothelium and Signaling Mechanisms," Stroke, Vol. 35, No. 11, 2004, pp. 2708-2711. doi:10.1161/01.STR.0000143319.73503.38

[36] A. Zacco, J. Togo, K. Spence, A. Ellis, D. Lloyd, S. Furlong, et al., "3-Hydroxy-3-Methylglutaryl Coenzyme a Reductase Inhibitors Protect Cortical Neurons from Excitotoxicity," Journal of Neuroscience, Vol. 23, No. 35, 2003, pp. 11104-11111. 